\title{
Pathologic Study of a Fatal Case of Dengue-3 Virus Infection in Rio de Janeiro, Brazil
}

\author{
C.A. Basílio-de-Oliveira ${ }^{1}$, G.R. Aguiar ${ }^{3}$, \\ Baldanza M.S. ${ }^{3}$, O.M. Barth ${ }^{2}$, W.A. Eyer-Silva ${ }^{1}$ \\ and M.V.Paes ${ }^{2}$
}

\author{
${ }^{1}$ Gaffrée and Guinle Teaching Hospital, Federal University of \\ Rio de Janeiro; ${ }^{2}$ Virology Department, Oswaldo Cruz Institute, \\ Fiocruz-RJ; ${ }^{3}$ São Vicente Hospital; Rio de Janeiro, RJ, Brazil
}

\begin{abstract}
Dengue hemorrhagic fever (DHF) is a severe febrile disease, characterized by abnormalities in hemostasis and increased vascular permeability, which in some cases results in hypovolemic shock syndrome and in dengue shock syndrome. The clinical features of DHF include plasma leakage, bleeding tendency and liver involvement. We studied the histopathological features of a fatal case of dengue-3 virus infection. The patient, a 63-year old male, presented with an acute onset of severe headache, myalgia and maculopapular rash. Tissue fragments (liver, spleen, lung, heart, kidney and lymph nodes) were collected for light microscopy studies and stained by standard methods. Histopathology revealed severe tissue damage, caused by intense hemorrhage, interstitial edema and inflammation. Some tissue sections were also processed with the immunoperoxidase reaction, which revealed the dengue viral antigen. Dengue-3 virus was isolated and identified with electron microscopy in a $\mathrm{C6} / 36$ cell culture inoculated with the patient's serum. Viral particles were detected in the infected cell culture.

Key Words: Dengue virus serotype 3, dengue hemorrhagic fever, immunoperoxidase.
\end{abstract}

Dengue hemorrhagic fever (DHF) is a severe febrile disease, characterized by abnormalities in hemostasis and by increased vascular permeability, which in some cases results in hypovolemic shock syndrome and dengue shock syndrome (DSS) [1]. The characteristics of DHF/DSS are perivascular edema, without destruction of the vascular endothelium, liver parenchyma necrosis and spleen, lymph node, and thymus cell hyperplasia [2]. Clinical features of DHF include plasma leakage, bleeding tendency, liver involvement, pleural or other effusions, hypoalbuminemia and hypoproteinemia [3]. The liver may be enlarged and serum activity of aminotransferases is usually increased [4]. In studies

Received on 28 June 2005; revised 10 August 2005.

Address for correspondence: Dr. Carlos Alberto Basílio-deOliveira. Serviço de Anatomia Patológica, Hospital Universitário Gaffrée Guinle. Rua Mariz e Barros, 775, Zip code: 20270-004 Rio de Janeiro / RJ, Brazil. E-mail: basiliopatologia@br.inter.net.

The Brazilian Journal of Infectious Diseases 2005;9(3):341-347 (C) 2005 by The Brazilian Journal of Infectious Diseases and Contexto Publishing. All rights reserved. of fatal dengue virus (DENV) infection, the DENV antigen was detected in the cytoplasm of phagocytic mononuclear cells of various tissues, including the liver, spleen, brain and lung [5]. Serology and detection of DENV serotype 3 by the polymerase chain reaction technique (PCR) have been used to establish a postmortem diagnosis of hepatic failure secondary to DHF [6]. Pathological findings reported in patients with DSS include liver centrilobular necrosis, changes in fatty tissue, Kupffer cell hyperplasia, acidophilic bodies, and monocyte infiltration of the portal tracts [2,7]. In fatal cases, DENV-antigen-positive hepatocytes were seen surrounding areas of liver necrosis [8-10]. Information about the target tissues and cells suitable for viral infection has been derived from examination of blood samples and tissues collected at autopsy. DENV antigens have been observed both in Kupffer cells and in hepatocytes [11]. Strong tissue alterations associated with DHF/DSS are observed in the liver, bone marrow, lymphoid organs, heart, lungs and kidneys [2]. The activity of serum aminotransferases was found to be significantly greater in patients with DHF/DSS than in those with DF [12]. 
In the early months of 2002, the state of Rio de Janeiro was severely burdened by a large-scale dengue epidemic [13, 14]. In contrast to the 1986-1987, 19901991 and 1995 epidemics, the 2001-2002 epidemic was characterized by the circulation of serotype 3 , which had been isolated for the first time in Brazil a few months earlier $[15,16]$. We report the findings of a virological and pathological post-mortem evaluation of a fatal case of dengue- 3 virus infection in an inhabitant of the city of Rio de Janeiro, who died during the 2001/ 2002 epidemic [13].

\section{Case Report}

The patient, a 63-year old retired male, who lived in the Mangueira neighborhood, presented with an acute onset of severe headache, myalgia, anorexia, abdominal pain, maculopapular rash, and petechial hemorrhage. Leukopenia, hemoconcentration and thrombocytopenia were evident on peripheral blood examination. Four days later, diarrhea and hemoptysis ensued. Increased serum activity of aspartate aminotransferase (75IU/L), creatine phosphokinase (126IU/L), myoglobin (174ng/mL), amylase (411IU/ L) and lipase (41 IU/L) were recorded, as well as a prolonged prothrombin time $(35 \%)$. An echocardiogram showed global dysfunction of the left ventricle, with an ejection fraction of $53 \%$. Dengue IgM and IgG antibody assays were both reactive. A progressively deteriorating clinical picture of shock syndrome and acute pulmonary congestion soon followed. The patient died, with a clinical diagnosis of dengue hemorrhagic fever, heart failure, acute abdomen and pancreatitis.

\section{Morphology and immunostaining of tissues}

Tissue fragments (liver, spleen, lung, heart and kidney) were fixed by immersion in Millonig's fixative and processed for hematoxylin and eosin (HE) staining, and sections were processed by immunoperoxidase reaction, using a monoclonal DENV-3 antibody.
Detection of dengue- 3 virus in C6/36 cell line culture inoculated with patient serum

The blood was centrifuged at $400 \mathrm{rpm}$ for 5 minutes, and the serum was collected and inoculated (100PL) into a monolayer of $\mathrm{C} 6 / 36$ cell culture. After inoculation for 1 hour at $28^{\circ} \mathrm{C}$, monolayers were grown in L-15 medium supplemented with $1 \%$ non-essential amino acids, $10 \%$ tryptose phosphate broth and $10 \%$ fetal bovine serum. The tubes were observed daily for viral cytopathic effects during 15 days. A negative control was prepared, using a non-infected monolayer of C6/ 36 cell culture. Presence of viral antigen in C6/36 cells was achieved by the indirect immunofluorescence technique, using a type-specific monoclonal antibody for DENV serotype 3 (3H5) (ATCC).

\section{Transmission electron microscopy}

Monolayers of infected and control C6/36 cell cultures were fixed in $1 \%$ buffered glutaraldehyde, dehydrated and embedded in epoxy resin for electron microscope observations. Ultra-thin sections $50-70 \mathrm{~nm}$ thick were obtained using a diamond knife, double stained with uranyl acetate and lead citrate and observed with a Zeiss EM-900 transmission electron microscope.

\section{Results}

Multiple purpuric and petechial hemorrhagic lesions were evident, especially around needle puncture sites. Hemorrhages were also present in serous cavities, including hemorrhagic pleuritis and pericarditis. The liver and spleen were grossly congested with multiple hemorrhagic foci, and a perforated duodenal ulcer was seen (Figure 1). On optical microscopy, there was a mononuclear cell infiltrate around the portal space, with focal vacuolization in the cytoplasm of hepatocytes. Hepatic sinusoids were infiltrated and free collagen filaments and hemorrhagic congestion were observed (Figures 2a and b). Generalized vascular damage was seen in the spleen, with hemorrhage, increased vascular 
Figure 1. A: hemorrhage of the great epiploon and hemorrhage in the serous of the intestinal tract. B: liverhepatic congestion (arrow) on the liver surface covered by sharp exudate as a result of perforation into duodenal ulceration (arrow).C and D: heart. hemorrhagic (arrow) fibrin pericarditis. E: lung - hemorrhagic (arrow) sulphution of the visceral pleura. F: spleen - splenic congestion and hemorrhagic (arrows) capsular focus.
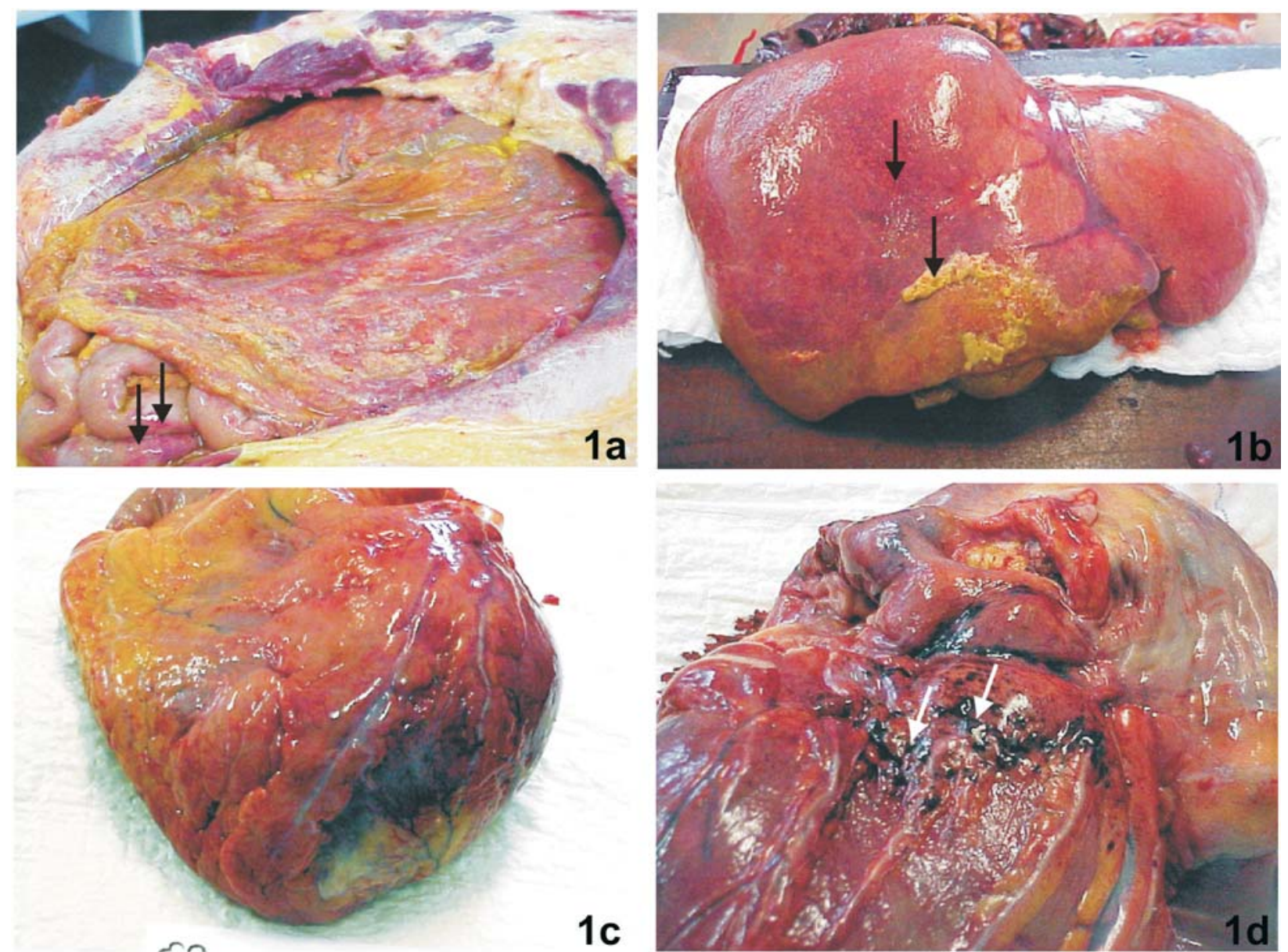

a
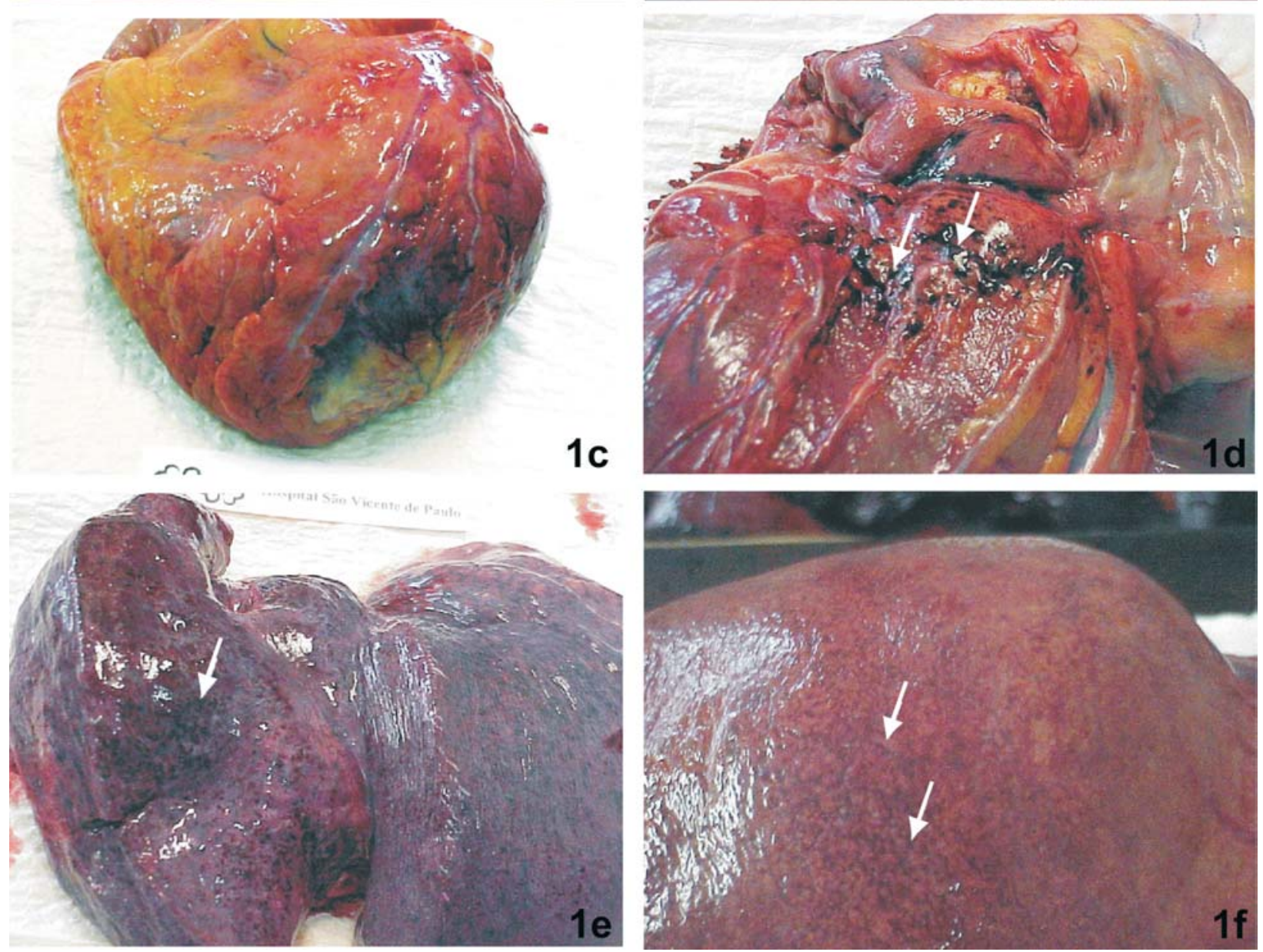
Figure 2. A and B: liver tissue. Increased inflammatory infiltrate and collagen filaments around the vessels can be noted in the portal space (PS). The sinusoidal capillaries show intense circulation of macrophage/lymphocytes (IC) causing injury of hepatocytes. Hematoxilin-eosin stain. Bar $=67 \mathrm{Pm}$ Bar $=43 \mathrm{Pm}$. C: immunoperoxidase study of liver tissue demonstrating viral antigen (asterisk) inside the cytoplasm of hepatocytes (H), Bar=20Pm. D and E: lung tissue. The intense infiltrate of alveolar macrophage/lymphocytes (AM) into the alveolar capillaries can be observed. Note the interstitial edema (IE) and hemorrhage (He) inside the alveolar space. Hematoxilin-eosin stain. Bar $=40 \mathrm{Pm}, \mathrm{Bar}=40 \mathrm{Pm}$. F: viral antigen demonstrated in the cytoplasm of pulmonary endothelial cells (EC) and alveolar macrophages (AM), Bar=24Pm. G: spleen tissue. Cell vacuolization and inflammatory cells (IC) of splenic sinusoids was verified in the red pulp. Interstitial edema (IE) and vacuolization occur in the splenic parenchyma. Haematoxilin-eosin stain. Bar $=22 \mathrm{Pm} 2 \mathrm{~h}$ Viral antigen present in the cytoplasm of inflammatory cells (IC) in the spleen, Bar=13Pm.
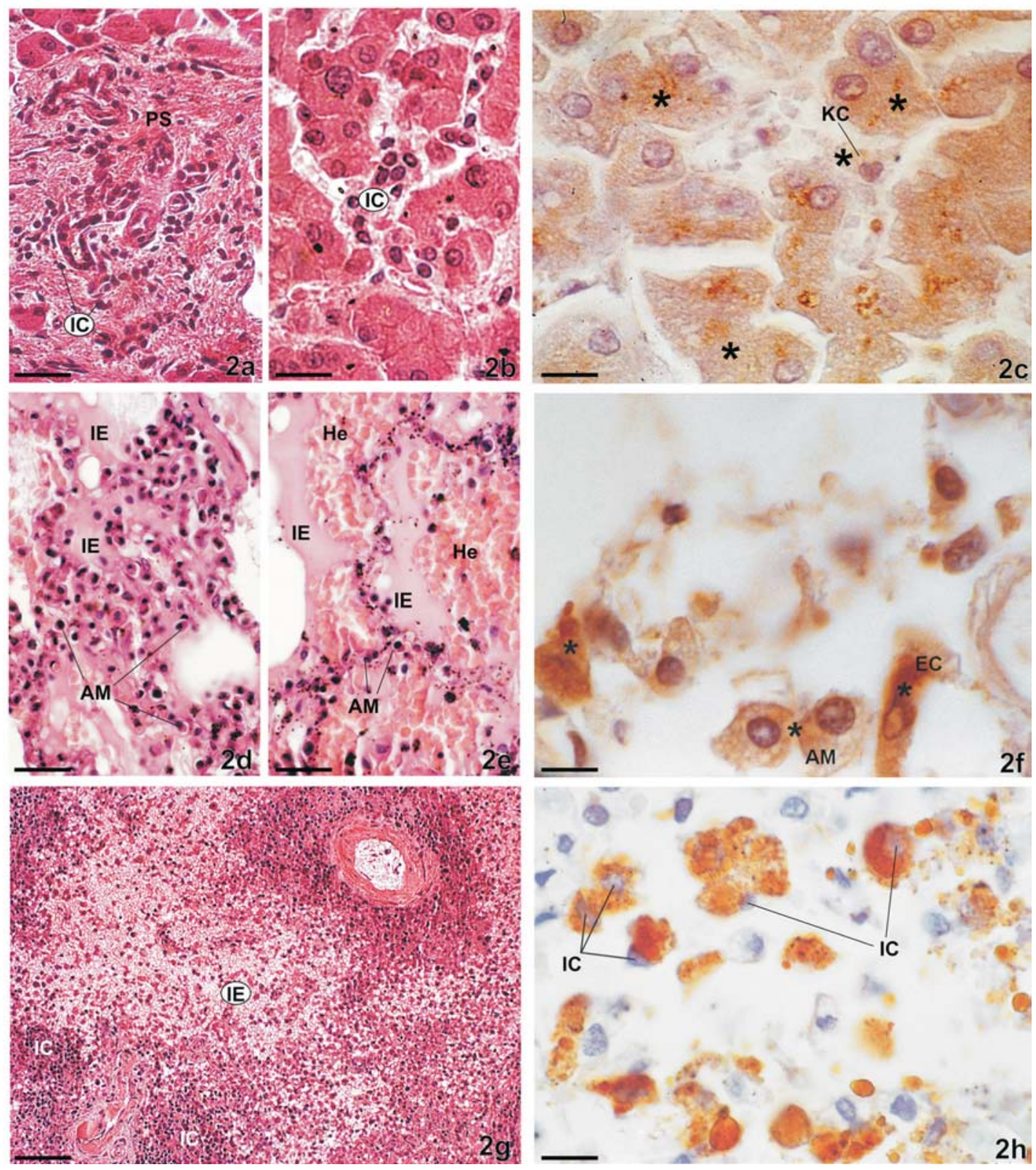

www.bjid.com.br 
edema and plasma leakage (Figure 2g). The lungs were diffusely infiltrated by mononuclear cells, with interstitial edema and diffuse alveolar congestion and hemorrhage. There was also hemorrhage into the great vessels (Figures 2d and e). Renal cortical sections showed hemorrhage on glomerular capillaries and proximal convoluted tubules. Renal medullary tissue also displayed a mononuclear infiltrate around the collecting ducts, with hemorrhagic foci, interstitial edema and vascular congestion (Figures $3 a$ and $b$ ).

Immunostaining of liver, spleen, lung, heart and kidney tissues, using the specific DENV-3 immunoperoxidase technique, disclosed the viral antigen on hepatocytes, Kupffer cells, alveolar macrophages, endothelial cells, and inflammatory cells in the medullar region of the kidney. DENV-3 virus particles were detected in the $\mathrm{C} 6 / 36$ cell culture inoculated with the patient's serum, under electron microscopy. Some vacuolated cells exhibited viral particles inside the cisternae of the rough endoplasmic reticulum(Figure 4).

\section{Discussion}

In the histopathological analysis of this fatal case of DENV-3 infection, we found severe damage to the vascular system, hepatic injury, and mononuclear cell infiltration in many target organs. Viral antigen was also demonstrated in inflammatory cells of the lung and spleen. We suggest that viral replication also occurs in macrophages and leukocyte cells. The autopsy showed numerous petechiae in the skin, with areas of hemorrhage. These data corroborate the conception that the liver is an important site of dengue virus replication. Intense injury to many organs was demonstrated, such as focal vacuolization of hepatocytes, and increasing concentrations of inflammatory cells inside sinusoid capillaries. This suggests the participation of cytokines and inflammatory mediators, causing alteration of vascular permeability. The lung and spleen also displayed focal vacuolization and interstitial edema around an infiltration of macrophages and lymphocytes. We found areas of intense parenchymal hemorrhage in many organs. Reported cases of dengue fever have detected DENV immunoreactivity in brain tissue cells, such as neurons, astrocytes, microglia and endothelial cells [19]. In a fatal case, vascular congestion and edema were especially conspicuous in the white matter and in mononuclear macrophage-like cells. Labeling of viral antigens were observed with light microscopy, within and outside small veins, many of them leading into brain tissue [5]. In our patient, viral antigen was found in inflammatory cells of the liver, lung, heart, kidney and lymph nodes, as well as in hepatocytes and endothelial cells. Similarly, dengue virus has previously been identified within liver tissue, and dengue virus antigen was identified within Kupffer cells of infected individuals [20]. Elevation of serum aminotransferase activity is almost universal in individuals with DHF [17]. The histopathology showed marked macrovesicular fatty changes, midzonal hepatocellular necrosis and focal lymphocytic and polymorphonuclear leucocyte infiltration in a case of dengue hemorrhagic fever with fulminant hepatic failure [6]. In our patient, hepatocellular injury was also observed, and focal microvesicular steatosis was found around the central lobular vein. The hepatic alteration consisted of diffuse necrosis of sinusoid capillaries, suggesting release of cytokines and activation of the complement system, as well as endothelial dysfunction. In conclusion, the patient in our study, infected with DENV-3, died with considerable hepatic injury, as well as interstitial edema and inflammatory infiltration in many organs.

\section{Acknowledgements}

We gratefully acknowledge José Farias Filho of the Laboratório de Flavivírus, Departmento de Virologia, Instituto Oswaldo Cruz, Bruno Eschenazi of the Laboratório de Produção e Tratamento de Imagens, Instituto Oswaldo Cruz and Aline Vilas Boas Vianna for technical assistance. We also thank Pedro Vasconcelos of the Instituto Evandro Chagas, Belém, for providing the DENV-3 antibodies. Financial support was provided by CAPES and CNPq. 
Figure 3. A, B and C: kidney tissue. The cortical region shows hemorrhage (He) in glomerulus capillaries and proximal convolute tubules. Note interstitial edema (IE) in both cortical and medullar region. Hematoxilin-eosin stain. Bar $=59 \mathrm{Pm}$ Bar=55Pm 3d) viral antigen inside inflammatory cells in the renal medullar region, Bar=12Pm.

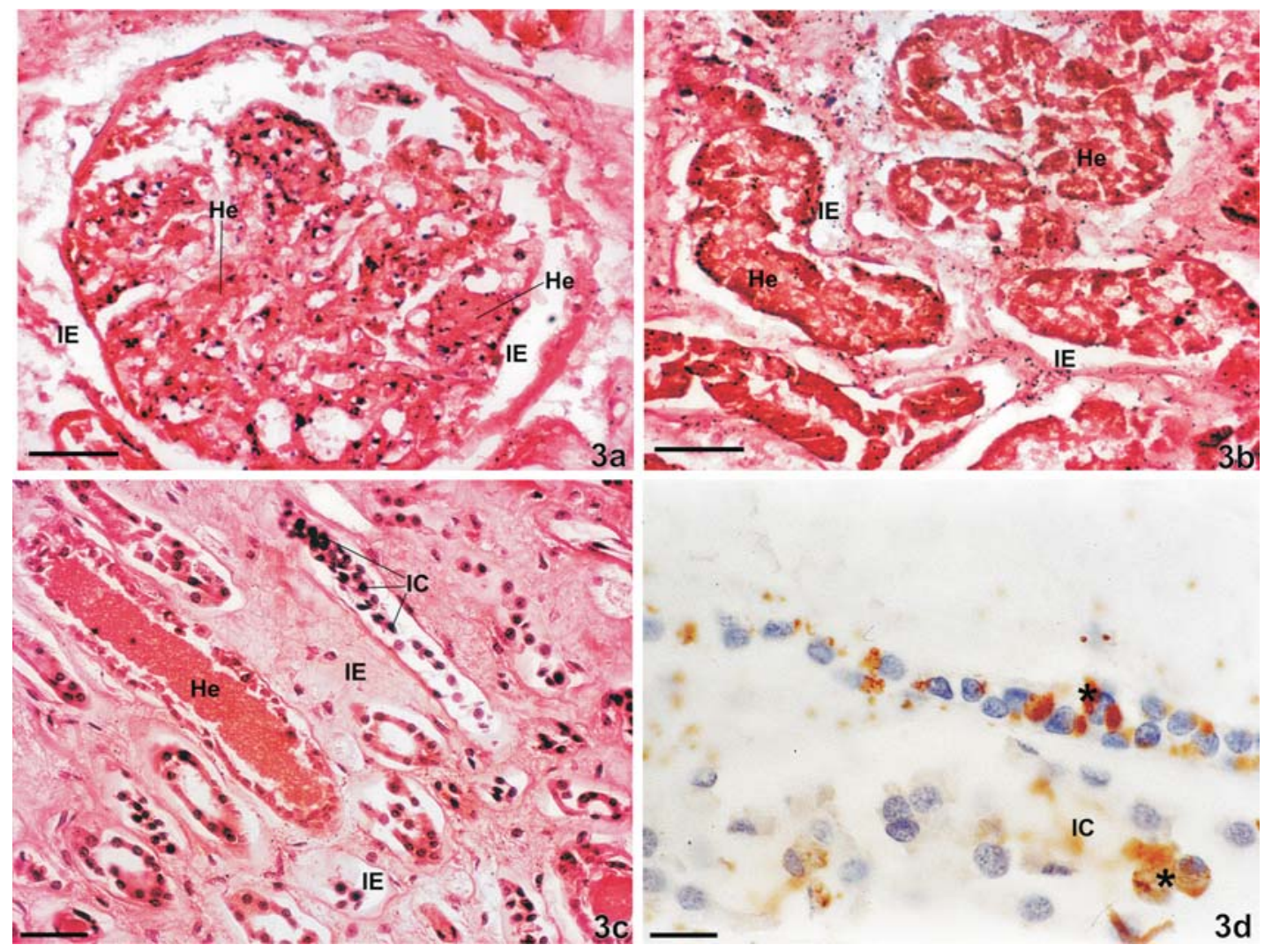

Figure 4. C6/36 cell monolayer culture inoculated with the serum of the dengue-3 virus infected patient. Viral particles (arrow) are located inside cisternae of the rough endoplasmic reticulum. $\mathrm{N}=$ nucleus. $\mathrm{M}=$ mitochondrion. Bar $=0.0003 \mathrm{Pm}$.

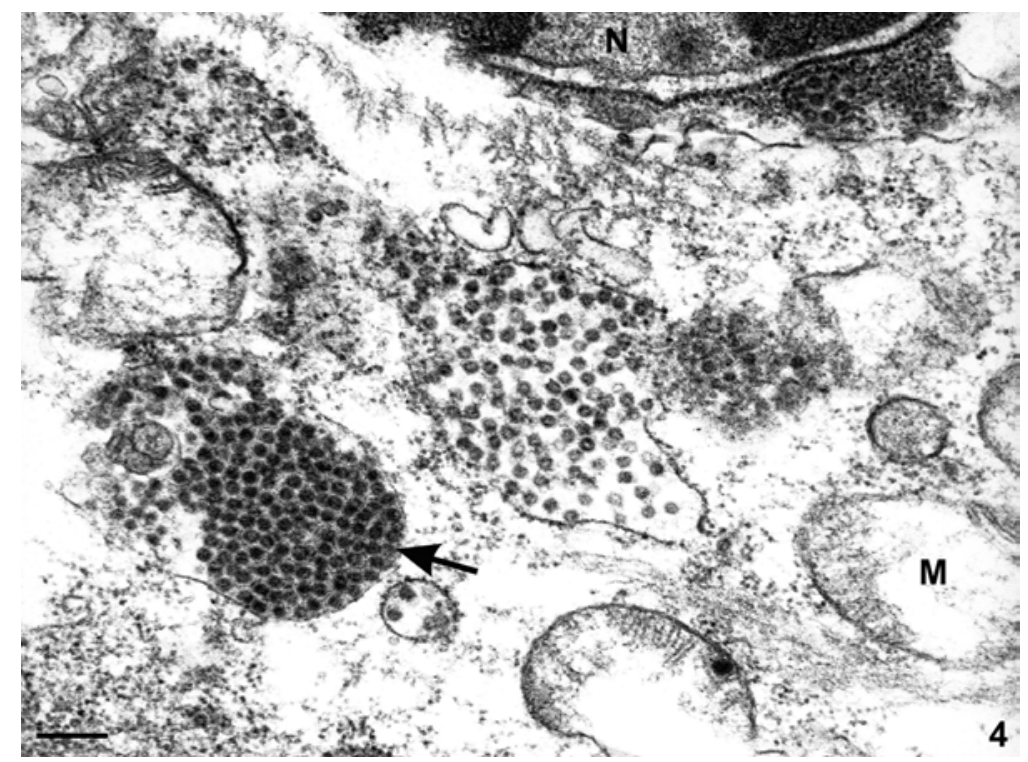




\section{References}

1. Halstead S.B. Observations related to pathogensis of dengue hemorrhagic fever. VI. Hypotheses and discussion. Yale J Biol Med 1970;42:350-62.

2. Bhamarapravati N., Tuchinda P., Boonyapaknavik V. Pathology of Thailand haemorrhagic fever: a study of 100 autopsy cases. Ann Trop Med Parasitol 1967;61:500-10.

3. Halstead S.B. Antibody, macrophages, dengue virus infection, shock, and hemorrhage: a pathogenetic cascade. Rev Infect Dis 1989; 11 :S830-9.

4. Kuo C.H., Tai D.I., Chang-Chien C.S., et al. Liver biochemical tests and dengue fever. Am J Trop Med Hyg 1992;47:265-70.

5. Miagostovich M.P., Ramos R.G., Nicol A.F., et al. Retrospective study on dengue fatal cases. Clin Neuropathol 1997; 16:204-8.

6. Lawn S.D., Tilley R., Lloyd G., et al. Dengue hemorrhagic fever with fulminant hepatic failure in an immigrant returning to Bangladesh. Clin Infect Dis 2003;37:e1-4.

7. Burke T. Dengue haemorrhagic fever: a pathological study. Trans R Soc Trop Med Hyg 1968;62:682-92.

8. Couvelard A., Marianneau P., Bedel C., et al. Report of a fatal case of dengue infection with hepatitis: demonstration of dengue antigens in hepatocytes and liver apoptosis. Hum Pathol 1999;30:1106-10.

9. Huerre M.R., Lan N.T., Marianneau P., et al. Liver histopathology and biological correlates in five cases of fatal dengue fever in Vietnamese children. Virchows Arch 2001;438:107-15.

10. Rosen L., Khin M.M. Recovery of virus from the liver of children with fatal dengue: reflections on the pathogenesis of the disease and its possible analogy with that of yellow fever. Res Virol 1989; 140:351-60.

11. Hall W.C., Crowell T.P., Watts D.M., et al. Demonstration of yellow fever and dengue antigens in formalin-fixed paraffin-embedded human liver by immunohistochemical analysis. Am J Trop Med Hyg 1991;45:408-17.

12. Kalayanarooj S., Vaughn D.W., Nimmannitya S., et al. Early clinical and laboratory indicato,rs of acute dengue illness. J Infect Dis 1997;176:313-21.

13. Casali C.G., Pereira M.R., Santos L.M., et al. [The epidemic of dengue and hemorrhagic dengue fever in the city of Rio de Janeiro, 2001/2002]. Rev Soc Bras Med Trop 2004;37:296-9.

14. Passos M.N., Santos L.M., Pereira M.R., et al. Clinical differences observed in patients with dengue caused by different serotypes in the epidemic of 2001/2002, occurred in Rio de Janeiro. Rev Soc Bras Med Trop 2004;37:293-5.
15. Rocco I.M., Kavakama B.B., Santos C.L. First isolation of dengue 3 in Brazil from an imported case. Rev Inst Med Trop Sao Paulo 2001;43:55-7.

16. Nogueira R.M., Miagostovich M.P., de Filippis A.M., et al. Dengue virus type 3 in Rio de Janeiro, Brazil. Mem Inst Oswaldo Cruz 2001;96:925-6.

17. Nguyen T.L., Nguyen T.H., Tieu N.T. The impact of dengue haemorrhagic fever on liver function. Res Virol 1997; 148:273-7.

18. Rosen L., Drouet M.T., Deubel V. Detection of dengue virus RNA by reverse transcription-polymerase chain reaction in the liver and lymphoid organs but not in the brain in fatal human infection. Am J Trop Med Hyg 1999;61:720-4.

19. Ramos C., Sanchez G., Pando R.H., et al. Dengue virus in the brain of a fatal case of hemorrhagic dengue fever. $\mathrm{J}$ Neurovirol 1998;4:465-8.

20. Guzman M.G., Kouri G. Dengue: an update. Lancet Infect Dis 2002;2:33-42. 